\title{
Effects of Repeated Intravitreal Injections of Dexamethasone Implants on Intraocular Pressure: A 4-Year Study
}

This article was published in the following Dove Press journal: Clinical Ophthalmology

\author{
Elena Pacella (1) \\ Lorenzo Loffredo iD ${ }^{2}$ \\ Mariaelena Malvasi ${ }^{\prime}$ \\ Edoardo Trovato Battagliola (iD) \\ Daniela Messineo iD ${ }^{3}$ \\ Fernanda Pacella' \\ Loredana Arrico id \\ 'Department of Sense Organs, University \\ of Rome La Sapienza, Rome, Italy; \\ ${ }^{2}$ Department of Clinical, Internal \\ Medicine, Anesthesiology and \\ Cardiovascular Sciences, University of \\ Rome La Sapienza, Rome, Italy; \\ ${ }^{3}$ Department of Radiological, Oncological \\ and Pathological Anatomy Sciences, \\ Sapienza University of Rome, Rome, Italy
}

Correspondence: Fernanda Pacella Department of Sense Organs, University of Rome La Sapienza, Policlinico Umberto I, Viale del Policlinico I55-0016I, Rome, Italy

Tel +39 3333534396

Email fernanda.pacella@gmail.com
Purpose: Dexamethasone and other corticosteroids are administered intravitreally to treat a variety of retinal diseases. As a side effect, they can alter intraocular pressure (IOP). The purpose of this study is to describe the incidence, severity, and management of ocular hypertension following the administration of multiple intravitreal injections of dexamethasone implants.

Materials and Methods: A total of 78 eyes of 78 subjects (males 62\%; females 38\%; mean age $67 \pm 13$ years SD) received a total of 152 intravitreal injections of $0.7 \mathrm{mg}$ dexamethasone implants over 4 years. Indications included retinal vein occlusion (87\%), diabetic macular edema (9\%), wet-type age-related macular degeneration (4\%). Ocular hypertension was defined as intraocular pressure above $23 \mathrm{mmHg}$ or any pressure increase of $10 \mathrm{mmHg}$ or more from baseline values. IOP was measured by applanation tonometry before the injection (T0), as well as one week (T1), one month (T2), and three months (T3) afterwards.

Results: Five percent (4/78) of subjects developed ocular hypertension after the 1st injection. On the second and third rounds, additional 7.2\% (3/42) and 4.2\% (1/24) of subjects developed the same side effect. Among the 8 subjects who received a fourth injection, none was found with OHT. Pressure elevations were detected at T2 and T3. In all patients, topical medical therapy was sufficient to lower the IOP below threshold. Mean pressure variations following the first injection as compared to previous recorded values were $+0.97 \mathrm{mmHg}$ (T1), $+0.92 \mathrm{mmHg}$ (T2), and $-0.41 \mathrm{mmHg}(\mathrm{T} 3)(\mathrm{p}<0.05)$. Mean pressure variations following the second injection were $+0.54 \mathrm{mmHg}$ (T1), $+0.23 \mathrm{mmHg}$ (T2) and $-0.66 \mathrm{mmHg}$ (T3) $(\mathrm{p}<0.05)$.

Conclusion: Ocular hypertension is a recognized side effect of intravitreal dexamethasone. Some patients develop it right after the first injection, while others develop it subsequently, on the 2 nd or 3 rd round. This side effect becomes most apparent 30-90 days following the implantation procedure and responds well to topical pressure-lowering medications.

Keywords: intravitreal dexamethasone implant, Ozurdex injection, hypertension, side effect, complications, retinal vein occlusion

\section{Introduction}

Intravitreal corticosteroids are routinely prescribed in clinical practice. They can be used as first-line medication or when other treatments fail. Common indications include refractory macular edema resistant to anti-VEGF therapy, ${ }^{1-6}$ retinal vein occlusion (RVO), ${ }^{7-9}$ and chronic non-infectious uveitis, ${ }^{10}$ among others.

Dexamethasone is one of the corticosteroids available for intravitreal use. It is injected in the form of a biodegradable implant that slowly releases $0.35 \mathrm{mg}$ or 0.7 $\mathrm{mg}$ of active drug into the vitreous over a period of about 6 months. ${ }^{11,12}$ However, 
this route of administration is associated with some side effects that include cataract formation and intraocular pressure (IOP) spikes. Ocular hypertension (OHT) can occur immediately after the injection due to volume expansion, or weeks to months later due to the steroid effects on aqueous drainage. ${ }^{13}$ Identifying and treating this side effect is important, as elevated IOP constitutes an important risk factor for primary open-angle glaucoma (POAG) incidence and progression. ${ }^{14}$

The effects of single intravitreal injections of dexamethasone implants on ocular pressure spikes have already been described in literature. The MEAD study showed that as many as $41.5 \%$ of patients receiving the $0.7 \mathrm{mg}$ implant and $37.6 \%$ of patients receiving the $0.35 \mathrm{mg}$ implant required pressure-lowering medications. ${ }^{15}$ The SAFODEX study showed similar results, with as many as $31 \%$ of eyes ultimately requiring pressure-lowering medications. ${ }^{16}$ In both studies, topical medications were generally effective in treating pressure spikes and incisional glaucoma surgery was performed in less than $1 \%$ of cases. In other studies, the incidence of this side effect was much lower. ${ }^{3,7,9,16-25}$ This probably depends on the threshold value set to define ocular hypertension and the population characteristics. ${ }^{26}$ Variables that increase the risk of secondary OHT include: pre-existing glaucoma, higher baseline IOP, younger age, previous episode of ocular hypertension following a steroid injection, uveitis, and higher steroid dose usage. ${ }^{27,28}$
In clinical practice, however, macular edema does not always resolve with a single injection and retreatment is often necessary. The effects of repeated injections on IOP have not been studied extensively. For this reason, authors provide additional data on the relationship between repeated intravitreal injections of dexamethasone implants and ocular pressure.

\section{Materials and Methods}

In this retrospective study, 78 eyes of 78 subjects (males $62 \%$; females 38\%; mean age 67 years \pm 13 SD as depicted in Figure 1) affected by variable degrees of macular edema caused by retinal vein occlusion $(87 \%)$, diabetic macular edema (9\%), and wet-type age-related macular degeneration (wet-AMD) (4\%) (Figure 2). In wet-AMD subjects, macular edema was unresponsive to anti-VEGF injections. All patients had within-range IOP upon enrollment. Two patients $(2.56 \%)$ were taking pressure-lowering medications for early-stage POAG.

\section{Ethical Approval}

This study protocol was approved by "La Sapienza, University of Rome", Ethics Board (Protocol No. 1076/14).

\section{Inclusion Criteria}

- Age > 18;

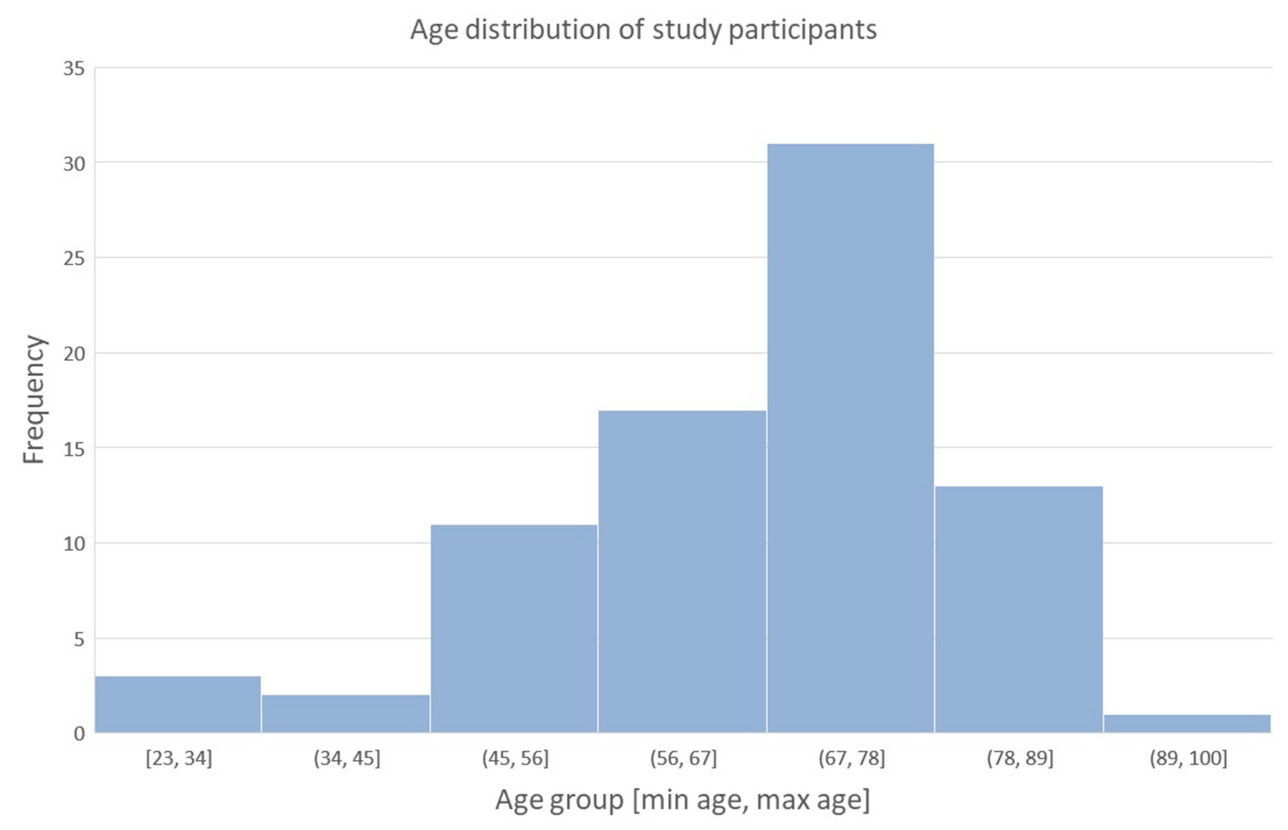

Figure I Age distribution of participants. Mean age $67 \pm 13$ years SD. 


\section{Retinal pathologies of study participants}

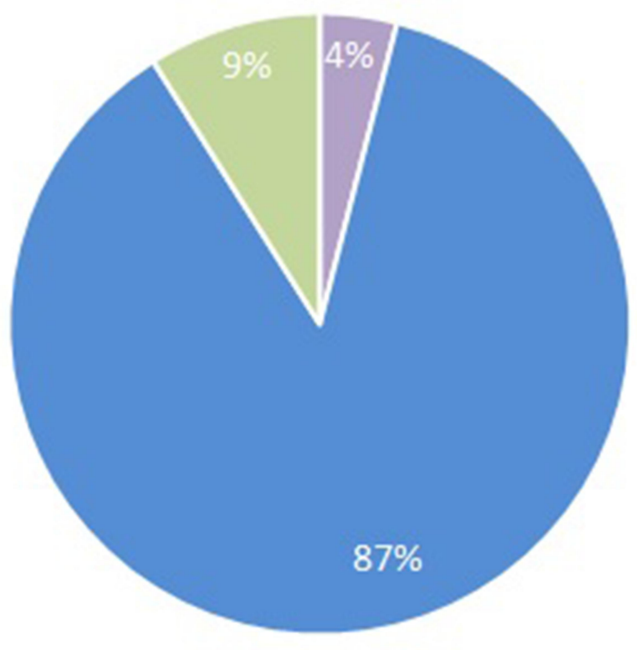

Wet-type AMD $\approx \mathrm{RVO}=\mathrm{DME}$

Figure 2 Retinal pathologies of enrolled subjects. Retinal vein occlusion (RVO) in $87 \%$ of subjects. Diabetic macular edema (DME) in $9 \%$ of subjects. Wet-type agerelated macular degeneration (Wet-type AMD) that was refractory to anti-VEGF therapy in $4 \%$ of subjects.

- Macular edema caused by retinal vein occlusion, wet-AMD, or diabetic retinopathy;

- $\mathrm{BCVA}<45$ letters ETDRS, equivalent to a LogMAR value between 1 and 0.2 ;

- Central macular thickness on OCT $\geq 285 \mu \mathrm{m}$;

- Treatment of naïve patients;

\section{Exclusion Criteria}

- Previous laser treatments or intravitreal procedures;

- Cataract;

- Glaucoma;

- Epiretinal membrane visible on OCT;

- Any ocular surgery performed within the previous 6 months;

- Pregnancy;

\section{Informed Consent}

All subjects signed written consent.

\section{First Assessment and Follow-Ups}

The following evaluations were performed upon enrollment:
- BCVA measurement on a decimal scale, as well as on ETDRS and logMAR scales;

- Intraocular pressure measurement with Goldmann applanation tonometer;

- Slit-lamp biomicroscopy of both anterior and posterior segments;

- Macular thickness evaluation with Heidelberg Spectralis SD-OCT, fast macula protocol;

Patients were re-evaluated one week (T1), one month (T2) and three months (T3) after each injection in the same fashion.

\section{Implantation Procedure}

The implant was injected in the inferotemporal vitreous cavity through the 22-gauge needle of the disposable device. Topical antibiotic/steroid medication was given for 3 days after the implant procedure.

\section{Definition of Ocular Hypertension}

Ocular hypertension was defined as intraocular pressure above $23 \mathrm{mmHg}$ or any pressure increase of $10 \mathrm{mmHg}$ or more from baseline values.

\section{Macular Thickness Evaluation with SD- OCT}

Macular scans were obtained using the fast macula protocol on the Heidelberg Spectralis SD-OCT. Automatic real time (ART) mean value of 9, which acquires 25 horizontal lines ( $6 \times 6 \mathrm{~mm}$ area), each consisting of 1024 A scans per line. The central retinal thickness (CRT) was defined as the distance between the inner limiting membrane to the outer border of the retinal pigment epithelium via the automatic segmentation algorithms.

\section{Statistical Analysis}

Data was collected using Microsoft Excel spreadsheets (Microsoft Excel 2010). Statistical analysis was performed using SPSS (IBM SPSS Version 20, IBM, New York, NY, USA). Statistical correlation was determined using the Friedmann test. A P-value $<0.05$ was considered significant.

\section{Results}

A total of 152 intravitreal injections were performed on 78 eyes. Forty-four (56\%) of these subjects received a second intravitreal injection. Twenty-four (31\%) received a third 
injection. Only $8(10 \%)$ received a fourth injection. Repeat injections were performed at least 3 months apart.

Ocular hypertension was defined as $>23 \mathrm{mmHg}$ or an increase of $10 \mathrm{mmHg}$ or more from baseline. All patients had within-range IOP upon enrollment. Two patients $(2.56 \%)$ were taking pressure-lowering medications for POAG.

As shown in Figure 3, around 5\% (4 out of 78), 7.2\% (3 out of 42), and 4.2\% ( 1 out of 24 ) of patients developed ocular hypertension after, respectively, the first, second, and third injection. The greatest pressure spikes were found 4-12 weeks following each procedure. The highest spike was $38 \mathrm{mmHg}$ recorded one month after the first injection. This represented an increase of $24 \mathrm{mmHg}$ from baseline. This was from a 23-year-old male diagnosed with BRVO, with the following IOP values: $14 \mathrm{mmHg}$ at baseline (T0), $16 \mathrm{mmHg}$ at $\mathrm{T} 1,38 \mathrm{mmHg}$ at $\mathrm{T} 2$, and $26 \mathrm{mmHg}$ at T3. This patient was prescribed hypotensive therapy at time $\mathrm{T} 2$.

Figure 4 shows mean, median, interquartile ranges at baseline and T1, T2, and T3 after each injection. Mean pressure changes after the first injection as compared to previous recorded value: $+0.97 \mathrm{mmHg},+0.92 \mathrm{mmHg}$, and $-0.41 \mathrm{mmHg}$ on $\mathrm{T} 1, \mathrm{~T} 2$, and $\mathrm{T} 3$, respectively $(\mathrm{p}<0.05)$. Mean pressure changes after the second injection as compared to previous recorded value: $+0.54 \mathrm{mmHg}$, $+0.23 \mathrm{mmHg}$ and $-0.66 \mathrm{mmHg}(\mathrm{p}<0.05)$.

Subjects who developed ocular hypertension (8 out of $78,10 \%)$ were prescribed pressure-lowering drops and all of them responded successfully to medical therapy. The hypotensive drops used were either of the following: timolol, brimonidine and timolol, tafluprost, or bimatoprost.

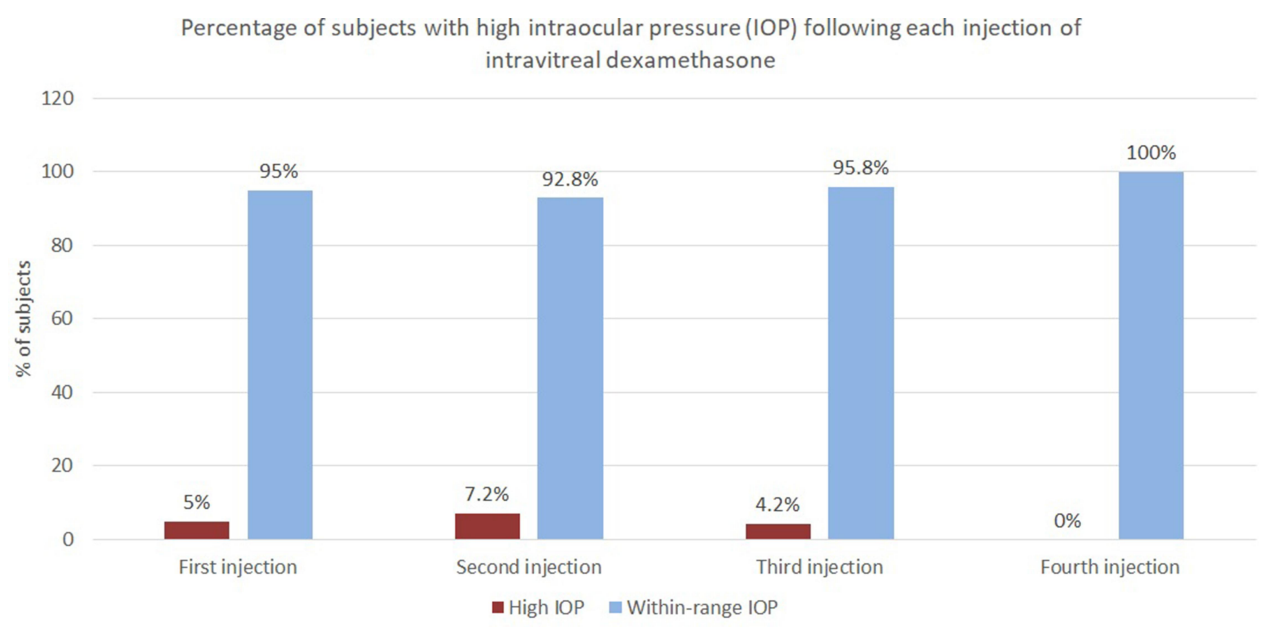

Figure 3 Percentage of subjects with high versus normal IOP after each injection.

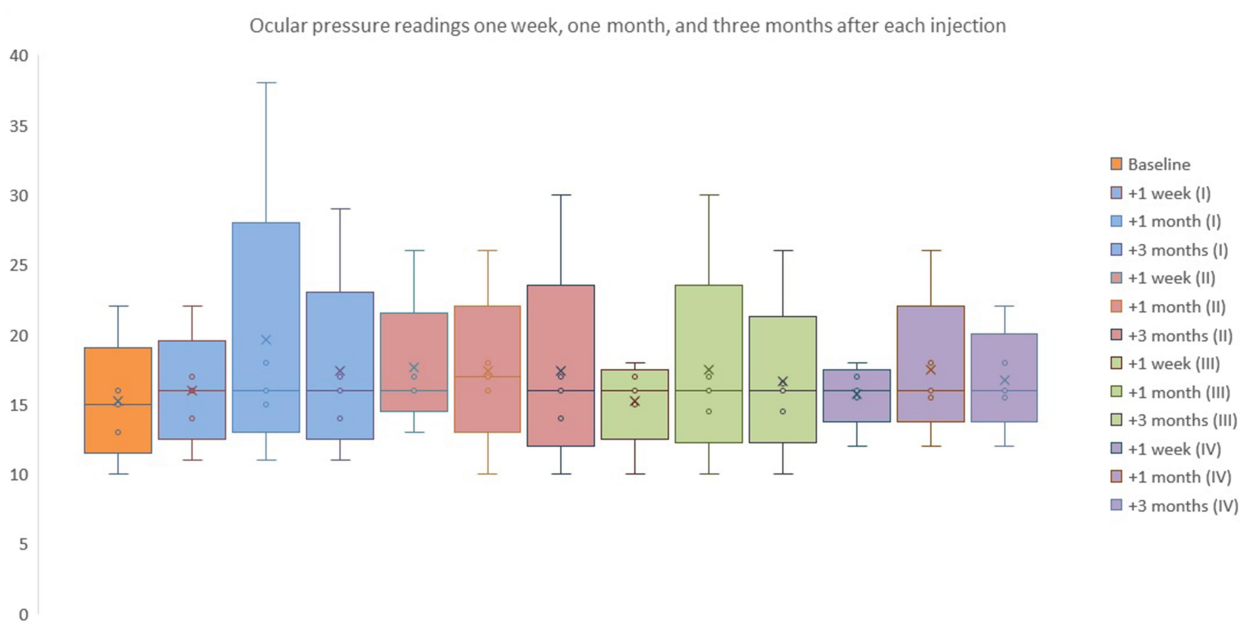

Figure 4 Median values and quartiles range at baseline (T0) and one week (TI), one month (T2), and three months (T3) after each injection. 
Choices were made on a case-by-case basis depending on the severity of ocular hypertension, as well as the patient's preferences and underlying systemic comorbidities. Subjects with ocular hypertension were kept on pressurelowering medications if retreatment was indicated. This explains a decreasing percentage of subjects found with ocular hypertension on subsequent injections, as shown in Figure 3. No glaucoma surgery or laser procedure was performed. IOP spikes did not affect visual acuity or visual fields. No RNFL thickness changes were noted on followup OCT RNFL exams.

The two subjects with POAG showed a mild IOP increase. Even if their IOP values remained below the chosen threshold of $23 \mathrm{mmHg}$, hypotensive therapy in these patients was stepped up to maintain their ocular pressure values similar to baseline.

\section{Discussion}

Previous studies have shown that common side effects of intravitreal dexamethasone implants include intraocular hypertension, cataract formation, and more rarely, anterior dislocation. ${ }^{29}$ The incidence of ocular hypertension varies significantly from study to study, from $6 \%$ to almost $50 \%{ }^{3,7,9,16-25}$ This difference in incidence probably depends on several factors, which include patients' characteristics, their pre-existing comorbidities, as well as the pressure threshold set to define ocular hypertension. In our study, the cumulative percentage of subjects who developed ocular hypertension after single or multiple dexamethasone injections was $10 \%$ (8 out of 78 ). RVO was the main indication (87\%). Table 1 shows a comparison with previous studies. In general, IOP elevation is transient and reaches peak values around 30-60 days following the injection. ${ }^{23}$ Topical medical therapy is almost always sufficient. ${ }^{15,16,30}$ Return to baseline values is expected within six months. ${ }^{26}$

In patients receiving multiple injections, some studies showed no correlation between the number of injections and the risk of developing ocular hypertension, ${ }^{19,31}$ while one study found an increased risk for IOP spikes below $30 \mathrm{mmHg} .{ }^{32}$ Our study showed a higher mean IOP after the second injection. This increase was low, but statistically significant $(\mathrm{P}<0.05)$. In addition, among patients

Table I Comparison with Previous Studies

\begin{tabular}{|c|c|c|c|c|}
\hline Study & $\begin{array}{l}\text { Number of } \\
\text { Eyes Treated }\end{array}$ & Indications & Definition of OHT & $\begin{array}{l}\text { Percentage of Participants } \\
\text { Who Developed OHT }\end{array}$ \\
\hline Chin et al $(2017)^{33}$ & 59 & $\begin{array}{l}\text { BRVO, CRVO, } \\
\text { uveitis, DME, CME }\end{array}$ & $\begin{array}{l}1 O P \geq 30 \mathrm{mmHg} \text { or an increase of } \geq \\
10 \mathrm{mmHg} \text { from baseline }\end{array}$ & $26.9 \%$ \\
\hline Malclès et al $(2017)^{16}$ & 421 & $\begin{array}{l}\text { RVO, DME, CME, } \\
\text { uveitis }\end{array}$ & $\begin{array}{l}\mathrm{IOP} \geq 25 \mathrm{mmHg} \text { or an increase of } \geq \\
10 \mathrm{mmHg} \text { from baseline }\end{array}$ & $28.5 \%$ \\
\hline Choi et al $(2019)^{23}$ & 540 & $\begin{array}{l}\text { RVO, DME, CME, } \\
\text { uveitis }\end{array}$ & $\begin{array}{l}\text { IOP } \geq 25 \mathrm{mmHg} \text { or an increase of } \geq \\
10 \mathrm{mmHg} \text { from baseline }\end{array}$ & $12.6 \%$ \\
\hline Mayer et al $(2013)^{21}$ & 64 & BRVO, CRVO & Increase of $5 \mathrm{mmHg}$ from baseline & $39.5 \%$ \\
\hline Bahadorani et al $(2018)^{32}$ & 183 & $\begin{array}{l}\text { BRVO, CRO, DME, } \\
\text { uveitis }\end{array}$ & $\mathrm{IOP} \geq 23 \mathrm{mmHg}$ & $31 \%$ \\
\hline Haller et al $(2010)^{31}$ & 841 & BRVO, CRVO & $\mathrm{IOP} \geq 25 \mathrm{mmHg}$ & $16 \%$ \\
\hline Hemarat et al $(2018)^{34}$ & 260 & $\begin{array}{l}\text { DME, BRVO, CRVO, } \\
\text { uveitis, others }\end{array}$ & $\mathrm{IOP} \geq 25 \mathrm{mmHg}$ & $26.2 \%$ \\
\hline Sudhalkar et al $(2018)^{35}$ & 378 & $\begin{array}{l}\text { DME, BRVO, CRVO, } \\
\text { CME }\end{array}$ & $\begin{array}{l}\text { IOP } \geq 25 \mathrm{mmHg} \text { or an increase of } \geq \\
10 \mathrm{mmHg} \text { from baseline }\end{array}$ & $23.27 \%$ \\
\hline Bakri et al $(2016)^{17}$ & 31 & BRVO, CRVO & $\mathrm{IOP} \geq 22 \mathrm{mmHg}$ & $45 \%$ \\
\hline Reid et al $(2015)^{19}$ & 61 & BRVO, CRVO & $\mathrm{IOP} \geq 25 \mathrm{mmHg}$ & $12 \%$ \\
\hline This study & 78 & $\begin{array}{l}\text { BRVO, CRVO, DME, } \\
\text { wet-AMD }\end{array}$ & $\begin{array}{l}1 O P \geq 23 \mathrm{mmHg} \text { or an increase of } \geq \\
10 \mathrm{mmHg} \text { from baseline }\end{array}$ & $10 \%$ \\
\hline
\end{tabular}

Abbreviations: OHT, ocular hypertension; IOP, intraocular pressure; BRVO, branch retinal vein occlusion; CRVO, central retinal vein occlusion; DME, diabetic macular edema; Wet-AMD, wet-type age-related macular degeneration; CME, cystoid macular edema. 
who did not experience IOP spikes after the first injection, some did after the second or third implantation. Pressure spikes responded well to topical medical therapy in all cases.

\section{Key Messages}

1. What is already known about this subject?

Steroids injected in the vitreous body of the eye can increase intraocular pressure in susceptible subjects. In some patients, this effect becomes apparent after multiple injections.

\section{What are the new findings?}

Subjects who did not develop ocular pressure spikes following the first injection are still at risk for ocular hypertension on subsequent injections.

3. How might these results change the focus of research or clinical practice?

Subjects who receive intravitreal dexamethasone should be always regularly monitored for ocular pressure spikes, regardless of their pre-injection ocular pressure or response to their first injection. With dexamethasone implants, the greatest pressure spikes can be generally expected one month following the injection.

Pressure spikes that develop on subsequent injections can be effectively managed with topical pressure-lowering medications.

\section{Disclosure}

The authors have no financial disclosure or conflicts of interest, direct or indirect, to declare, and they had full access to all data in the study.

\section{References}

1. Khan Z, Kuriakose RK, Khan M, Chin EK, Almeida DRP. Efficacy of the intravitreal sustained-release dexamethasone implant for diabetic macular edema refractory to anti-vascular endothelial growth factor therapy: meta-analysis and clinical implications. Ophthalmic Surg Lasers Imaging Retina. 2017;48:160-166. doi:10.3928/2325816020170130-10

2. Pacella F, Romano MR, Turchetti P, et al. An eighteen-month followup study on the effects of Intravitreal Dexamethasone Implant in diabetic macular edema refractory to anti-VEGF therapy. Int $J$ Ophthalmol. 2016;9:1427-1432.

3. Pacella F, Ferraresi AF, Turchetti P, et al. Intravitreal injection of ozurdex $\left({ }^{\circledR}\right)$ implant in patients with persistent diabetic macular edema, with six-month follow-up. Ophthalmol Eye Dis. 2016;8:1116. doi:10.4137/OED.S38028

4. Pacella E, La Torre G, Impallara D, et al. Efficacy and safety of the intravitreal treatment of diabetic macular edema with pegaptanib: a 12month follow-up. Clin Ter. 2013;164:e121-6.

5. Pacella E, Pacella F, La Torre G, et al. Testing the effectiveness of intravitreal ranibizumab during 12 months of follow-up in venous occlusion treatment. Clin Ter. 2012;163:e413-22.
6. Bianchi E, Scarinci F, Ripandelli G, et al. Retinal pigment epithelium, age-related macular degeneration and neurotrophic keratouveitis. Int J Mol Med. 2013;31:232-242. doi:10.3892/ijmm.2012.1164

7. Garweg JG, Zandi S. Retinal vein occlusion and the use of a dexamethasone intravitreal implant $\left(\mathrm{Ozurdex}^{\circledR}\right)$ in its treatment. Graefes Arch Clin Exp Ophthalmol. 2016;254:1257-1265.

8. Hahn P, Fekrat S. Best practices for treatment of retinal vein occlusion. Curr Opin Ophthalmol. 2012;23:175-181. doi:10.1097/ ICU.0b013e3283524148

9. Pacella F, La Torre G, Basili S, et al. Comparison between "early" or "late" intravitreal injection of dexamethasone implant in branch (BRVO) or central (CRVO) retinal vein occlusion: six-months follow-up. Cutan Ocul Toxicol. 2017;36:224-230. doi:10.1080/ 15569527.2016.1254648

10. Whitcup SM, Robinson MR. Development of a dexamethasone intravitreal implant for the treatment of noninfectious posterior segment uveitis. Ann N Y Acad Sci. 2015;1358:1-12. doi:10.1111/nyas.12824

11. Haghjou N, Soheilian M, Abdekhodaie MJ. Sustained release intraocular drug delivery devices for treatment of uveitis. J Ophthalmic Vis Res. 2011;6:317-329.

12. Chang-Lin J-E, Attar M, Acheampong AA, et al. Pharmacokinetics and pharmacodynamics of a sustained-release dexamethasone intravitreal implant. Invest Ophthalmol Vis Sci. 2011;52:80-86. doi:10.1167/iovs.10-5285

13. Taylor SRJ, Isa H, Joshi L, Lightman S. New developments in corticosteroid therapy for uveitis. Ophthalmologica. 2010;224(Supp1 1):46-53. doi:10.1159/000318021

14. Actis AG, Versino E, Brogliatti B, Rolle T. Risk factors for Primary Open Angle Glaucoma (POAG) progression: a study ruled in Torino. Open Ophthalmol J. 2016;10:129-139.

15. Boyer DS, Yoon YH, Belfort R Jr, et al. Three-year, randomized, shamcontrolled trial of dexamethasone intravitreal implant in patients with diabetic macular edema. Ophthalmology. 2014;121:1904-1914. doi:10.1016/j.ophtha.2014.04.024

16. Malclès A, Dot C, Voirin N, et al. Safety of intravitreal dexamethasone implant (OZURDEX): the SAFODEX study incidence and risk factors of ocular hypertension. Retina. 2017;37:1352-1359.

17. Bakri SJ, Omar AF, Iezzi R, Kapoor KG. Evaluation of multiple dexamethasone intravitreal implants in patients with macular edema associated with retinal vein occlusion. Retina. 2016;36:552-557. doi:10.1097/IAE.0000000000000750

18. Proença Pina J, Turki K, Labreuche J, Duhamel A, Tran THC. Efficacy and safety in retinal vein occlusion treated with at least three consecutive intravitreal dexamethasone implants. $J$ Ophthalmol. 2016;2016:6016491. doi:10.1155/2016/6016491

19. Reid GA, Sahota DS, Sarhan M. Observed complications from dexamethasone intravitreal implant for the treatment of macular edema in retinal vein occlusion over 3 treatment rounds. Retina. 2015;35:1647-1655. doi:10.1097/IAE.0000000000000524

20. Tservakis I, Koutsandrea C, Papaconstantinou D, Paraskevopoulos T, Georgalas I. Safety and efficacy of dexamethasone intravitreal implant (Ozurdex) for the treatment of persistent macular edema secondary to retinal vein occlusion in eyes previously treated with anti-vascular endothelial growth factors. Curr Drug Saf. 2015;10:145-151. doi:10.2174/1574886309666140805142245

21. Mayer WJ, Wolf A, Kernt M, et al. Twelve-month experience with Ozurdex for the treatment of macular edema associated with retinal vein occlusion. Eye. 2013;27:816-822. doi:10.1038/eye.2013.79

22. Mishra SK, Gupta A, Patyal S, et al. Intravitreal dexamethasone implant versus triamcinolone acetonide for macular oedema of central retinal vein occlusion: quantifying efficacy and safety. Int $J$ Retina Vitreous. 2018;4:13. doi:10.1186/s40942-018-0114-2

23. Choi W, Park SE, Kang HG, et al. Intraocular pressure change after injection of intravitreal dexamethasone (Ozurdex) implant in Korean patients. Br J Ophthalmol. 2019;103(10):1380-1387. doi:10.1136/ bjophthalmol-2018-312958 
24. Pacella E, Vestri AR, Muscella R, et al. Preliminary results of an intravitreal dexamethasone implant $\left(\mathrm{Ozurdex}^{\circledR}\right)$ in patients with persistent diabetic macular edema. Clin Ophthalmol. 2013;7:1423-1428. doi: $10.2147 /$ OPTH.S48364

25. Korobelnik J-F, Kodjikian L, Delcourt C, et al. Two-year, prospective, multicenter study of the use of dexamethasone intravitreal implant for treatment of macular edema secondary to retinal vein occlusion in the clinical setting in France. Graefes Arch Clin Exp Ophthalmol. 2016;254:2307-2318. doi:10.1007/s00417-016-3394-y

26. Pacella F, Turchetti P, Santamaria V, et al. Differential activity and clinical utility of latanoprost in glaucoma and ocular hypertension. Clin Ophthalmol. 2012;6:811-815. doi:10.2147/OPTH.S13777

27. Pacella E, Pacella F, Cavallotti C, Librando A, Feher J, Pecori-Giraldi $\mathrm{J}$. The combination latanoprost-timolol versus twice daily $0.50 \%$ timolol administration either associated or not with latanoprost: efficacy and tolerability in the primary open-angle glaucoma. Eur Rev Med Pharmacol Sci. 2010;14:477-480.

28. Feher J, Kovacs I, Pacella E, Keresz S, Spagnardi N, Balacco Gabrieli C. Pigment epithelium-derived factor (PEDF) attenuated capsaicin-induced neurotrophic keratouveitis. Invest Ophthalmol Vis Sci. 2009;50:5173-5180. doi:10.1167/iovs.08-1852

29. Pacella F, Agostinelli E, Carlesimo SC, et al. Management of anterior chamber dislocation of a dexamethasone intravitreal implant: a case report. J Med Case Rep. 2016;10:282. doi:10. 1186/s13256-016-1077-2
30. Bucolo C, Gozzo L, Longo L, Mansueto S, Vitale DC, Drago F. Long-term efficacy and safety profile of multiple injections of intravitreal dexamethasone implant to manage diabetic macular edema: a systematic review of real-world studies. $J$ Pharmacol Sci. 2018;138:219-232. doi:10.1016/j.jphs.2018.11.001

31. Haller JA, Bandello F, Jr BR, et al. Randomized, sham-controlled trial of dexamethasone intravitreal implant in patients with macular edema due to retinal vein occlusion. Ophthalmology. 2010;117:11341146.e3. doi:10.1016/j.ophtha.2010.03.032

32. Bahadorani S, Krambeer C, Wannamaker K, et al. The effects of repeated Ozurdex injections on ocular hypertension. Clin Ophthalmol. 2018;12:639-642. doi:10.2147/OPTH.S148990

33. Chin EK, Almeida DRP, Velez G, et al. Ocular hypertension after intravitreal dexamethasone (Ozurdex) sustained-release implant. Retina. 2017;37(7):1345-1351. doi:10.1097/IAE.0000000000001364

34. Hemarat K, Kemmer JD, Porco TC, Eaton AM, Khurana RN, Stewart JM. Secondary ocular hypertension and the risk of glaucoma surgery after dexamethasone intravitreal implant in routine clinical practice. Ophthalmic Surg Lasers Imaging Retina. 2018;49(9):680-685. doi:10.3928/23258160-20180831-05

35. Sudhalkar A, Kodjikian L, Chhablani J, Bhojwani D, Vasavada A. Intraocular dexamethasone implant position in situ and ocular hypertension. Retina. 2018;38(12):2343-2349. doi:10.1097/IAE.0000000000001 883
Clinical Ophthalmology

\section{Publish your work in this journal}

Clinical Ophthalmology is an international, peer-reviewed journal covering all subspecialties within ophthalmology. Key topics include: Optometry; Visual science; Pharmacology and drug therapy in eye diseases; Basic Sciences; Primary and Secondary eye care; Patient Safety and Quality of Care Improvements. This journal is indexed on PubMed
Dovepress

Central and CAS, and is the official journal of The Society of Clinical Ophthalmology (SCO). The manuscript management system is completely online and includes a very quick and fair peer-review system, which is all easy to use. Visit http://www.dovepress.com/ testimonials.php to read real quotes from published authors. 